\title{
68. ON THE ORIGIN OF COMETS AND THEIR IMPORTANCE FOR THE COSMOGONY OF THE SOLAR SYSTEM
}

\author{
V. G. FESENKOV \\ Committee on Meteorites, U.S.S.R. Academy of Sciences, Moscow, U.S.S.R.
}

\begin{abstract}
The processes in interstellar space, as well as the structure of nebulae - including the numerous small condensations around newly formed stars - suggest a relationship to the formation of the cometary cloud surrounding the Sun. Study of carbonaceous chondrites has revealed that in the earliest stage of the solar system's existence frequent periods of brief heating occurred, leading to the formation of very complex organic compounds and also of chondrules of crystalline structure. This was most probably a consequence of cometary collisions. The Tunguska phenomenon illustrates some consequences of a similar collision.
\end{abstract}

It is appropriate to consider that comets, as well as stars, are formed in interstellar space. This is, however, a very complicated magnetohydrodynamical problem. The magnetic field in our Galaxy is strong enough to confine cosmic rays and, in all probability, to determine the structure of the galactic spiral arms. Interstellar space, highly heterogeneous in composition, is constantly being affected by the galactic nucleus; shock waves from supernova explosions are propagated in it, sudden compressions of matter occur, there is interaction between its dust and gas constituents and a great amount of turbulence. All this results in the formation of heterogeneities of various dimensions: globules occurring only in the nebulae, the development of Herbig-Haro objects (star-like condensations) and stellar chains and associations that rapidly disperse into space. Such stellar chains can be observed, for instance, in the filamentary nebulae of Cygnus that formed at the boundary between dust clouds as the result of a supernova explosion about $70000 \mathrm{yr}$ ago.

Of great interest is the Orion nebula, which surrounds an unstable system of stars in the Orion Trapezium that developed some $23000 \mathrm{yr}$ ago. This nebula has been found to consist mainly of a large number of heterogeneities containing almost all the mass. Attempts are made to find, by analysis of spectroscopic data, the relative velocities of heterogeneities similar to comets. In that same nebula there were recently discovered, in infrared light, protostars at the beginning of collapse.

The chemical composition of comets fully corresponds to this concept of their origin. According to spectral data cometary heads contain molecular compounds such as $\mathrm{C}_{2}, \mathrm{C}_{3}, \mathrm{CH}, \mathrm{NH}, \mathrm{CH}_{2}, \mathrm{OH}, \mathrm{CO}_{2}, \mathrm{NH}_{2}$, to which correspond the parent molecules $\mathrm{HCN}, \mathrm{NH}_{3}, \mathrm{CH}_{4}, \mathrm{C}_{2} \mathrm{H}_{2}$ and others; the composition of meteors suggests that silicates and various compounds involving heavy elements should also be included. The same compounds are found in interstellar space, this being compatible with the assumption of the development there of unstable bodies such as comets by the same processes connected with the formation of stars.

V. S. Safronov has expressed a similar viewpoint in developing his cosmogonical theory for the formation of the planets. He believes that the growth of the giant planets was accompanied by the gravitational ejection of part of their embryo material 
beyond the solar system, this process having led to the formation of an extended cometary cloud. He supposes that the total mass ejected constitutes from one-third to one-half of the mass of the giant planets (Safronov, 1969). This is equivalent to assuming that the planets developed through the successive combination of heterogeneities of cometary type and fairly complicated composition.

On the other hand, S. K. Vsekhsvyatskij has quite a different concept of the origin of comets - by cosmic vulcanism. His ideas are based in essence only on the supposition that in the inner regions of the solar system the short-period comets break up very rapidly, within a few centuries. Without going into details, we shall say that his concept cannot be accepted for a number of reasons. Firstly, it is quite impossible to produce by ejection from the planets the vast majority of comets in nearly parabolic orbits, which form a more or less uniform cloud in the vicinity of the Sun, extending according to J. H. Oort and G. A. Chebotarev - for about 150000 AU. Secondly, the highly unstable cometary matter is characteristic of the properties, not of planetary interiors, but of interstellar matter. Moreover, as Vsekhsvyatskij himself points out, a cosmic volcano calls for an unusually powerful source of energy, greatly exceeding that of nuclear reactions, and it could not possibly develop in bodies as small as planets - and especially their satellites.

Let us now dwell on some processes which occurred during the earliest stage of development of the solar system, prior to the formation of planets. Interesting data on the subject are supplied by a particular variety of meteorites - the carbonaceous chondrites. These objects retain an abundance of rare gases that corresponds to their cosmic abundance, and they are believed to be the oldest known matter. They are an amazing combination of organic hydrocarbon and nitrogen compounds and chondrules - small crystalline silicate balls. A detailed laboratory investigation of meteorites such as Orgueil, Cold Bokkeveld, and Murray has shown them even to contain such complex compounds as $\mathrm{C}_{6} \mathrm{H}_{5}\left[\mathrm{CH}\left(\mathrm{CH}_{3}\right)_{2}\right] ; \mathrm{C}_{6} \mathrm{H}_{4}\left(\mathrm{C}_{2} \mathrm{H}_{3}\right)_{3}$, anthracene, phenanthrene and other compounds up to the bases of DNA. This once gave rise to the belief that these meteorites or their parent bodies could earlier have carried life. However, detailed experiments (by Studler, Hayatsu, Anders, and others) have shown that these complex compounds may be reproduced with great accuracy under conditions of thermodynamic equilibrium in Fisher-Tropsch reactions from a medium of typically cometary composition but much lower hydrogen content. Meteoric matter provides a catalyst, and rapid heating to about $800-1000{ }^{\circ} \mathrm{C}$, followed by sudden cooling, is imperative. The formation of chondrules from the same material evidently requires much greater local heating, also followed by cooling.

Such bursts, which occurred during the preplanetary stage of the solar system, have been explained in various ways, e.g., by flares of the Sun itself before it joined the main sequence. In the opinion of H. C. Urey, mutual collisions of comparatively loose matter in a weak gravitational field generate a great deal of heat; many molten droplets are produced, and after rapid cooling they become chondrules. It is precisely this kind of process that would have occurred very frequently in the earliest stage of existence of a protoplanetary nebula that contained a large number of condensations of cometary type. On their mutual collision, accompanied by heating with subsequent 
expansion and rapid cooling, there would have resulted both complex organic compounds and, in similar quantities, chondrules. Together with other mineral matter, the chondrules subsequently formed more compact bodies - chondrites - and the latter gradually became part of much larger bodies. For a considerable length of time they experienced fairly complicated metamorphosis, accompanied by the formation of meteorites of different types. Such processes would most naturally occur in mutual collisions between comets - bodies of comparatively negligible masses, low density, and abundance of the simplest organic compounds with the inclusion of gases and meteoric matter, but naturally with a low hydrogen content.

The Tunguska meteorite, which was actually a small comet of minimum mass $10^{12}$ $\mathrm{g}$, can be cited as an example illustrating what happens when a cometary nucleus is suddenly decelerated. The tail of the comet interacted with upper layers of the Earth's atmosphere and caused a short-lived anomalous luminosity of the night sky. When the nucleus of the Tunguska comet reached an altitude of approximately $10 \mathrm{~km}$ above the Earth's surface it produced a tremendous explosion, the shock waves from which felled the taiga forest for hundreds of kilometres around. We have shown that this corresponds to an average density of the nucleus equal to about $0.1 \mathrm{~g} \mathrm{~cm}^{-3}$. It is known that material from the nucleus spread after the explosion over almost the whole Northern Hemisphere and considerably diminished the solar radiation received. A large number of silicate and magnetite balls, strongly resembling chondrules, fell on the ground in the vicinity of the epicentre, while along the whole trajectory of the bolide there was noted for the next few decades a considerable acceleration in the growth of vegetation; this was quite independent of the changes in the ecological conditions and probably connected with the precipitation of complex organic compounds.

We should point out that the above concept of the cometary origin of meteorites has also been suggested by other authors from other data. Yokoyama et al. (1968) have remarked that the asteroidal nature of meteorites does not conform well to their chemical and mineralogical composition; they emphasized, in particular, the absence in meteoritic matter of long-period isotopes formed by slow neutrons. Still earlier, Öpik (1966) discussed cometary nuclei as the principal source of meteorites. By comparing the orbits of comets, meteorites, and the asteroids of the Apollo group, Öpik concluded that all these bodies have a common origin, the comets being the initial bodies.

It thus seems most natural to assume that the planetary bodies formed from the heterogeneities of a protoplanetary nebula of cometary type. It was conventionally assumed that the protoplanetary nebula consisted of gas and dust of approximately the interstellar composition and that the dust particles gradually agglomerated into bodies of planetary size. This is, however, highly improbable. T. Gold believes that the supposed process of cementation of fine particles through their mutual collisions is the principal cosmogonical problem requiring solution. Urey also points out that there should be some forces, either electrostatic or ferromagnetic, to cement these particles together. Therefore he, and also Kuiper, prefer to believe that the protoplanetary nebula was divided in the course of fragmentation into a number of gas-dust masses or protoplanets. In these primary objects there were first formed small solid planets 
similar to the Moon in size, and they later combined into the present planets as the result of impact phenomena. The development of comogonical ideas increasingly leads to the conclusion that the comets - the heterogeneities contained in the protoplanetary nebula from the very beginning - were responsible for the complete evolution of the solar system and directly led to the formation of the planets.

\section{References}

Öpik, E.: 1966, Adv. Astron. Astrophys. 4, 301.

Safronov, V. S.: 1969, Ehvolyutsiya Doplanetnogo Oblaka i Obrazovanie Zemli i Planet., Nauka, Moscow, p. 186.

Yokoyama, Y., Mabuchi, H., and Labeyrie, J.: 1968, Symposium on Origin and Distribution of the Elements, Paris, p. 445. 